\title{
Lapurdum
}

Euskal ikerketen aldizkaria | Revue d'études basques |

Revista de estudios vascos | Basque studies review

$20 \mid 2017$

Numéro $X X$

\section{La place du Bordelais dans les relevés de Gerhard Rohlfs sur les spécificités du gascon}

\section{Alain Viaut}

\section{OpenEdition}

\section{Journals}

Édition électronique

URL : https://journals.openedition.org/lapurdum/3615

DOI : 10.4000/lapurdum.3615

ISSN : 1965-0655

\section{Éditeur}

IKER

Édition imprimée

Date de publication : 1 janvier 2017

Pagination : $303-316$

ISBN : 978-2-95534-135-3

ISSN : $1273-3830$

Référence électronique

Alain Viaut, «La place du Bordelais dans les relevés de Gerhard Rohlfs sur les spécificités du gascon », Lapurdum [En ligne], 20 | 2017, mis en ligne le 01 janvier 2021, consulté le 03 septembre 2021. URL : http://journals.openedition.org/lapurdum/3615; DOI : https://doi.org/10.4000/lapurdum.3615 


\section{La place du Bordelais dans les relevés de Gerhard Rohlfs sur les spécificités du gascon}

Alain VIAUT

CNRS-IKER

Louvrage Le gascon: études de philologie pyrénéenne, du linguiste allemand Gerhard Rolhlfs (1892-1986) s'inscrit dans une série d'études sur les substrats et les particularités linguistiques du gascon ${ }^{1}$, que l'accent soit mis sur sa participation foncière à l'ensemble occitan ou bien sur ses caractéristiques susceptibles de l'avoir résolument spécifié depuis ses origines comme irréductible sous-ensemble roman autonome au sein du groupe occitano-roman. $\mathrm{Si}$, dans ces deux cas, sa perception comme expression linguistique indépendante n'est que rarement postulée, son intégration à l'ensemble d'oc comme «occitan extrême» (Sauzet 2006) ou son articulation comme composante intrinsèque sont examinées à l'aide des indispensables analyses descriptives (Chambon 2003). Afin de compléter l'évocation partielle des travaux évoqués, nous renverrons également à la synthèse de X. Ravier sur le sujet (Ravier 1991, 8591).

La question de l'identité du gascon ne relève pas pour nous de la réalité constatée d'une distance linguistique avec les autres composantes de l'occitan. Celle-ci existe, et l'étude de ce qu'il en est à l'origine (Chambon \& Greub 2002 ; Dinguirard 1977) concerne son histoire propre comme, au-delà parfois, celle de l'occitan car - et Rohlfs lui-même le remarque à plusieurs reprises - parmi les faits qui la déterminent en font partie certains, structuraux,

1. Sur la question des substrats du gascon (aquitano-euskariens, ibères et autres), on mentionnera par exemple la contribution de Joaquín Gorrochategui sur les études de portant sur cette question autour des «langues anciennes des Pyrénées dans le sillage de Gehard Rohlfs» (Gorrochategui s. d.). 
en particulier quant à sa physionomie phonétique, qui peuvent se retrouver ailleurs, en Pays d'oc ou bien plus loin (Sauzet 2004, 19-20). Certains de ses traits identificateurs se sont étendus au-delà de son aire constitutive, ou bien aussi sont les héritiers de différents substrats pré-romans, de type globalement non indo-européen, et, plus précisément aussi, de type aquitano-euskarien², et celtique gaulois parfois. Le lexique est ici particulièrement convoqué, Ses aires sont répandues sur des espaces qui peuvent s'inscrire à peu près à l'intérieur des délinéaments isoglossiques du gascon comme les excéder. Il en va ainsi d'une aire qui va du nord de l'Espagne inclus jusqu'à la Garonne pour un certain nombre d'entre eux, pas forcément d'origine aquitano-euskarienne, qui contribuent à la caractériser et à laquelle G. Rohlfs se réfère notamment, de fait ou explicitement.

La question de la linguification ou non du gascon ne nous occupe pas ici. Elle est généralement argumentées par ses promoteurs par des faits de distance linguistique connus de la communauté scientifique qui les inclut néanmoins dans des appréhensions il est vrai soit globalisantes soit contrastées d'une matière d'oc («l'occitan et le gascon» dans une présentation souvent duelle chez les romanistes) qui est en même temps abordée, non moins généralement, comme un objet sociolinguistique unique. La question de l'identité du gascon relèverait d'abord, à notre sens, d'une dynamique proprement sociolinguistique reliée à l'existence d'un consensus social. Bien que localement (tendance présente en Béarn ${ }^{3}$ ) exprimée, nous ne voyons pas celle-ci pas se dégager de façon plus significative que d'autres tendances centrifuges au sein de la plupart des langues et inhérentes à leur vie. Par ailleurs, sans chercher à contribuer à la clôture de ce débat, il nous paraît en ressortir que l'occitan, d'un point de vue descriptif,

2. «la gasconité maximale n'est pas au centre de la Gascogne, disons dans l'Armagnac par exemple, mais aux marges. Cela s'accorde bien avec l'idée fondamentale que la Gascogne est un FarWest occitan ou un finisterre roman. Cela n'exclut pas, et cela est parfois établi, que le gascon conserve des mots ou des traits issus du substrat aquitain ou du voisinage du basque. Mais il semble que la physionomie du gascon doit beaucoup à sa position au bout du domaine occitan, à un des bouts du chemin de la romanisation. Il en résulte un mélange de conservations, d'innovations parfois débridées et de déphasages qui font que ce langage que d'autres Occitans (grammairiens et médiévaux il est vrai, les auteurs des leys d'Amors) ont dit «estranh», est un étranger du dedans, un estrangier del dedins, un estrangèr deu dehens, fait parfois de matériau familier» (Sauzet 2004, 21),

3. Un résultat de l'enquête sociolinguistique réalisé en 2008 par la Région Aquitaine sur l'occitan (Région Aquitaine 2009) indique que : «70 \% des personnes ayant déclaré connaître ou comprendre quelques éléments au moins de langue ont accepté que, pour la suite du questionnaire, cette langue soit dénommée «occitan». Pour les 30 \% restant, la langue a continué d'être dénommée selon le terme qui avait leur préférence (patois, béarnais, gascon, ...). Parmi ceux qui déclaraient connaître ou parler quelques éléments de la langue et la dénommaient «patois», l'acceptation du terme «occitan» pour la suite de l'enquête était de $62 \%$. Ce taux d'acceptation était de 60 \% pour ceux utilisant la dénomination «gascon» et de 59 \% pour ceux utilisant la dénomination «béarnais».» (Région Aquitaine 2009, 12). En ce qui concerne la dénomination «béarnais», cela reviendrait par exemple à considérer que les $53 \%$ des déclarants qui s'identifièrent à elle dans les Pyrénées-Atlantiques représenteraient à peu près 22 \% du sous-échantillon global de ce département. 
est soit considéré comme un continuum cohérent dès le moyen âge (Sauzet 2006), soit comme le résultat d’un agrégation «médiévale... et post-médiévale» ${ }^{4}$, étant entendu qu’il participe objectivement, depuis cette époque, quoiqu'avec ses traits propres, de la dynamique générale de l'aire linguistique d'oc ou occitane.

En attendant, l'ouvrage de G. Rohlfs sur le gascon est souvent cité pour étayer la spécificité du gascon que nul ne nie. Il sera intéressant de se reporter, à ce sujet, au compterendu de lecture de la première édition de 1935 qu'en fit Jean Bouzet (1935, 79-85). C'est en spécialiste tant de la matière gasconne que de l'hispanique qu'il fit une critique fine regrettant que la phonétique historique occupe une place trop minoritaire dans l'ouvrage, et mettant en relief l'intérêt par exemple d'aller au-delà de l'aire de référence largement pyrénéenne privilégiée par Rohlfs, en notant nombre de faits coïncidant entre le gascon et les domaines asturo-léonnais (surtout l'asturien occidental avec la palatalisation de -ll- intervocalique latin, similaire à l'aboutissement du même à [t $[$ ] [tj] puis [t] en finale masculine) et galaïco-portugais (ainsi, par exemple, de la chute de -n- intervocalique). Il prend une position prudente quant à la difficile et délicate question des substrats D’un côté, il admet la réalité de ceux qui sont d'origine aquitano-ibérique et se retrouvent en commun avec le basque connu. D’un autre côté, il apporte des informations supplémentaires utiles au réexamen de certaines étymologies, et, sans nier le grand intérêt de la synthèse de Rohlfs, invite à une recherche plus approfondie sur la configuration et les développements de la romanisation de la large zone concernée, basque comprise.

Létude de G. Rohlfs sur le gascon concerne ainsi surtout le lexique dont il vise à établir sa part d'originalité due, pour une part significative, au facteur géographique isolant: la fameuse boucle de la Garonne et, on l'oublie, le plus grand estuaire d'Europe où séjournent encore les énormes esturgeons européens (jusqu'à 500 kilos) sans parler des maigres de 100 kilos qui viennent s'y reproduire. Quant aux Pyrénées, on retrouve là cette question de la perméabilité des frontières orographiques tant il est vrai que les fleuves constituent peut-être d'autant plus des frontières que les zones économiques de vie dont ils sont constitutifs sont justement des zones à défendre et qui, par conséquent, sont susceptibles de faire frontière. Face à ces évocations un peu extra-scientifiques, l'ouvrage de Rohlfs ne penche pas résolument vers l'exotisme des substrats mais prend le risque de se livrer à une recension de ce qui serait constitutif de l'originalité du gascon en mettant l'accent sur les liens, certes, avec des substrat disons à rapprocher du basque connu, et sur la composition autonome d'un stock lexical relativement propre, du Nord de l'Aragon, notamment, au nord du domaine gascon que ceuxci soient d'origine pré-latine, gauloise ou latine.

Sans aller jusqu'à nous interroger ici sur le champ gradient de la gasconité cartographié

4. «Les dialectes ou variétés fondamentales de l'occitan ne sont pas le fruit de la segmentation interne progressive d'un introuvable proto-occitan, mais bien des données primitives. [...]. En d'autres termes, la structuration géographiques de la langue d'oc ne résulte pas de divisions dialectales internes successives, mais procède d'agrégations d'espaces inertes face aux domaines linguistiques voisins innovateurs. [...] On voit mieux par là, peut-être, pourquoi l'histoire de la lengo nostro est restée dans une large mesure, jusqu'à nos jours, l'épopée d'une conscience en quête d'identité.» (Chambon 2003, 47). 
dans l'Atlas linguistique et ethnographique de la Gascogne (Séguy VI, 2531), nous noterons seulement que les calculs dont il résulte le concentrent à la fois vers les Pyrénées et vers le sud-ouest du domaine, en laissant apparaître les trois pôles d'irradiation - principalement Bordeaux, Toulouse, Bayonne - du langage codifié d'oc, relié aux formes cultes latines puis dominantes françaises. Simplement, «les étapes du recul de l'ancienne langue indigène de l'Aquitaine» (Rohlfs, 38) - l'aquitain supposé avoir été proche de l'ancêtre du basque actuel correspondent à l'idée présente chez Rohlfs selon laquelle :

«ll ne faut pas oublier que le latin était la langue d'un peuple de la plaine qui vivait sous un
climat bien différent de celui des Pyrénées. on comprend bien que les bergers des montagnes,
étant les formes spéciales de leur vie quotidienne, possédaient dans leur langage bien des mots
qui n'avaient pas d'équivalent en latin et qui ne pouvaient être traduits en latin» (Rohlfs, 38).

Notre propos n'étant pas de remettre en cause cette assertion reposant sur le postulat classique du refuge montagnard, nous remarquons juste que les bergers ont aussi existé dans ladite plaine gasconne au sens large, comme une base économique et culturelle primordiale, jusqu'à la lande médoquine, au nord, avec un mode de vie également «spécial» et ancien. Les travaux du folkloriste landais Félix Arnaudin (1844 - 1921) en ont largement témoigné. Et puis les bergers ne sont pas forcément les seuls à perpétuer éventuellement des formes linguistiques anciennes. Au-delà, l'idée développée ici ne s'emploiera pas à faire assaut de gasconité en mettant en doute ces affirmations mais tentera une autre approche de la nature de cette dernière, centrée ici sur le lexique, lui-même principalement examiné par Rohlfs, sans a priori géolinguistique, en allant précisément revoir, après l'ALG et Rohlfs, ce qu'il en est à l'un des opposés de la banane épicentrique habituellement invoquée.

Nos observations prendront alors en compte de façon délibérée le nord du domaine gascon et seront basées principalement sur le Médoc au sens large, le «triangle médocain» selon l'approche de Pierre Bec (1974, 280-283), finalement représentative d'une bonne partie du Bordelais, le Bazadais se trouvant dans une situation de contact avec le gascon central notamment. Pour cela, nous nous réfèrerons d'abord aux données de l'Atlas linguistique et ethnographique de la Gascogne (ALG), à nos propres enquêtes linguistiques depuis la fin des années 1970 ainsi qu'au lexique inédit de François Conord (1882-1973) du parler basmédoquin (Conord < Viaut 1999). Rohlfs fait surtout appel dans ses relevés au Dictionnaire de S. Palay (1974), de l'école Gastou Febus, qui contient aussi des données médoquines pas toujours fiables. De façon secondaire, nous nous réfèrerons aussi à la zone immédiatement contiguë au sud et, en outre, linguistiquement la plus proche, principalement à des matériaux contenus dans des lexiques gascons du Pays de Buch (Moureau 1870 ; Vidal 1999 et 2002).

Nous laisserons de côté le début de l'ouvrage sur les origines historiques du gascon. La toponymie, en particulier, ne présente pas dans notre aire septentrionale un profil très original. Les toponymes suffixés avec -os y sont présents même jusqu'à la commune du Verdon-sur-Mer avec le quartier des Arros, et la carte de la page 31 au maillage très large ne saurait signaler tous les lieux-dits formés ainsi. Des données retenues par Rohlfs après, nous nous appuierons sur celles qui figurent essentiellement dans les chapitres II, III et IV.

Le chapitre II, intitulé «Les origines linguistiques préromanes» (dont gauloise), envisage tout d'abord les «Débris préromans dans le vocabulaire» (pp. 38-39) et le «Vocabulaire pyrénéen» avec quelques généralités dont on retrouvera plus loin des détails: 
«Ce sont des éléments légués aux parlers romans dans une époque plutôt récente et dont on retrouvera et dont il est assez facile de déterminer la source basque moderne» (Rohlfs 1977, 38). Parmi ces éléments, dont certains sont strictement liés à la flore pyrénéenne, des mots tels que eslurra ${ }^{5}$ 'glisser' et herping 'aiguille' connaissent des équivalents en Médoc (cf. infra). Les aires de certains de ces mots dépassent le territoire de l'Aquitaine antique (artigo 'friche', esquèr 'gauche', mano 'femelle stérile', etc.). De cette façon, Artiga et mana sont effectivement répandus dans notre aire bordelaise.

Les sous-chapitres qui suivent fournissent des liste de mots dont le caractère préroman s'entend au sens plein et ne renvoient pas seulement au basque, dont on doit s'interroger sur sa place en tant que point de repère pour les substrats ou de fournisseur de mots d'emprunt éventuellement ancien, mais aussi au gaulois, voire à des substrats pré-indo-européens plus diffus

Nous reprenons les intitulés de ces sous-chapitres ainsi que les numéros suivis des entrées de chaque notice.

\section{Noms de plantes (Rohlfs, 40-45)}

24: Pour herping 'aiguilles de pin', nous avons grepin (ALG IV, 1366) Haut-Médoc tandis que hurpin et herpin (ALG IV, 1366) en Bas-Adour. On trouve aussi grepin et gurpin, synonymes comme en Médoc (avec garbach et garbalh + barba de pin au nord), de garbalhs/ garbalhas 'aiguilles de pins sèches sur le sol' en Pays de Buch (Vidal 1999 : 64). Le basque erpin 'sommet, extrémité, pointe' est évoqué.

26 : Pour jaugue 'ajonc', dans les Landes, nous avons jauga, en Haut-Médoc et ajauga en Bas-Médoc. Le gaulois *jouga (FEW V, 51) est évoqué.

30 : Pour layà 'gaule de châtaignier' (Palay), le basque lai 'tige', 'branche' est avancé et le FEW convoqué (FEW V, 131). Ce rapprochement nous paraît contredit par les attestations flaja/flage (+ flauge/flauja) 'baguette, scion, ...', en languedocien (Alibert 1966) et par flage ou flaje 'baguette, houssine, ...', en gascon (Palay). Les équivalents eslaje, eslauje et leurs dérivés eslajàt -ade, eslajerade, -tade (Palay) sont donnés pour venir plus sûrement du latin flagellum'. La notation phonétisante du dictionnaire de S. Palay transcrit simplement une des prononciations de <lhaja>, <hlajat>. Flaja et hlajat, prononcés ['flaju] et [la'jat] au nord et au centre, et ['flazə] au sud, sont répandus en Médoc et désigne la branche de vigne de l'année. Le mot basque lai a peut-être été emprunté au gascon et non l'inverse. On notera simplement que, pour «fléau» (lhagèth), dans l'ALG (II, 296), Urt et Labastide Clairence, en contact avec l'aire du basque, donnent tous deux [la'jet].

33 : Landes sigorre 'espèce de jonc. Le mot sigòrra/sigòrla, répandu en Médoc, désigne le choin marisque. Le basque zigor est convoqué (voir aussi FEW XX, 18).

5. Les mots en gascon donnés par Rohlfs, qui sont généralement issus du dictionnaire de S. Palay, sont écrits à l'aide de la graphie de l'école félibréenne Gastou Febus. Nos transcriptions des mots que nous apportons le seront à l'aide de la graphie dite «occitane» ou «normalisée». 


\section{Noms d'animaux (Rohlfs, 45-48)}

42 : Gasc. lagàst 'tique'. LALG $(I, 63)$ atteste que ce mot est répandu dans toute l'aire gasconne. En Médoc, la forme regach [rre'yats] [rre'yat]] est générale, avec lagas au sud. En outre, une spécialisation, relevée par l'ALG, y demeure courante : regach (générique) + biuch [biưuts], [biựtS] (gonflée de sang) + à Saint-Yzans, peligre (tique blanche). Le lien est fait avec le basque lakasta, lakats.

43 : Gasc. lagagno 'tique', dont on retiendra cependant non ce sens mais celui de «chassie». Laire de laganha couvre toute l'aire gasconne (ALG IV, 1457), jusqu'en domaine languedocien (Alibert 1966). Il est présent en Médoc et dans l'Entre-Deux-Mers avec, également les variantes leganha et liganha. Le mot basque lakain, ayant la même signification, est invoquée.

47 : Pour le petit lézard gris, qui a fait l'objet d'une carte dans l'ALG (I, 38), Rohlfs donne plusieurs mots (chichanglo, sangaline, sicoulane, etc.) qui paraissent partir d'une forme commune. Et il les met en rapport avec ls formes basques sugelinda, sugalinda et sugandela. Dans le Médoc apparaissent (ALG I, 38) : sanguineta, à Cissac et Castelnau, singuineta, à Lacanau, et sangoina, au sud, à Saint-Jean d'Illac et Pessac, le tout en même temps ou à côté d'une aire angròta (Bas-Médoc, Entre-Deux-Mers et Dordogne rive droite).

\section{Terminologie pastorale (Rohlfs, 48-51)}

49 : Gasc. amoùrrou 'animal (mouton ou brebis) qui a le tournis'. Si morre, -a, morraud, $-a$, ont le sens de sot, morre a aussi celui d'engourdi (pour les doigts, les membres, les abeilles). La forme imorre, - a a été relevée à Saint-Laurent-Médoc (enq. A. Viaut, 1974) avec la même signification que celle retenue par Rohlfs.

56: Gasc. mano 'femelle stérile', dont l'aire dépasse largement le cadre gascon à l'intérieur de l'occitan en parallèle avec mando, mandu en basque, de sens similaire. Le Médoc utilise aussi le mot mana, et connait le concurrent mula, présent en oc sur une vaste aire.

57 : Gasc. mar, màrrou, ... 'bélier'. Ce mot, dont Rohlfs trouve le frère en basque, est général en Médoc (marre) et l'est aussi jusqu'en Provence et en Espagne. Lauteur évoque le mot basque marro 'mugissement du bœuf et du taureau' qui nous rappelle le verbe maonar (ou mahonar) en Médoc (nord et centre), avec l'hypothèse d'un amuïssement de -rr- comme dans le doublet, attesté, charrin/chaïn (ruissellement), également dans la même aire médoquine.

61 Gasc. segàlh 'agneau', est renvoyé au basque sehaildu 'maigrir'. Nous nous contenterons d'évoquer sans conviction, en Médoc, secalh/secalha (Viaut 1992, 216) qui désigne l'échalas de réforme ramassé pour être brûlé. Malgré la dérivation possible à partir de l'adjectif sec (< latin siccus) + suffixe péjoratif -alh, ledit échalas réformé est aussi bien sec que pourri ou ramolli lorsqu'il est apte à porter ce nom.

\section{La configuration du terrain (Rohlfs, 51-55)}

64 : Béarn artico, 'terre défrichée', largement répandu en toponymie dans l'aire occitane et le nord-ouest de l'Espagne, se trouve, de fait, également en Bordelais. Rohlfs présente des hypothèses sur l'origine discutée de ce mot, ou gauloise ou aquitano-pyrénéenne, et penche pour un lien avec le basque arte 'chène vert'. 
68: Gasc. eslurrà 'glisser', cartographié dans l'ALG (III, 816). Le Médoc présente une aire compacte avec lurjar [lyr'dza] [lyr'za] (ALG III, 816 : St-Vivien-Médoc, St-Yzans-Médoc, Hourtin), attesté aussi à Saint-Laurent-Médoc et Listrac (enq. A. Viaut). Cette aire médoquine est séparée d'une plus grande, sud-ouest, bornée par Mézos au nord, Labrit et Geaune à l'est, et par Lourdes et Arrens au sud-est. Pour l'origine du terme, Rohlfs suggère un croisement entre les mots basques lerratu 'glisser' et lurra 'la terre', ce qui supposerait une base lur (terre) présente (substrat ?) jusqu'au nord du domaine gascon.

70 : Gasc. gàngo 'crête de montagne'. Cela nous renvoie au basque gangar (crête de coq), retenu par le FEW (XX, 18 : cf. gàngo : crête de montagne in Aure, Palay). En Médoc, le mot ganga (Avensan, enq. A. Viaut 1973) a le sens de dent de râteau de fenaison. Par ailleurs, le mot gam (ALG 2 307) a été en usage dans tout le Médoc, avec, en sus, gam/pua à SVivien, dent/brin à Lacanau, et dent à Pessac), présente une aire unique avec cette base en gascon.

71 : Gasc. gàrro 'rocher'. Peut-être en lien à travers la motivation de dureté, le mot garrau du centre et du nord du Médoc désigne le mâchefer, et le dérivé garravar [garra'wa], l'endroit où il abonde dans les antiques lieux où l'on fondait le fer local. Notons que le toponyme Gar(r)os/Gar(r)osse, signalé pour comporter la racine pré-indoeuropéenne gar/kar (cf. infra), se rencontre en plusieurs endroits du Bordelais. Rohlfs invoque pour cette entrée tant le basque harri/*karri qu'une base pré-indoeuropéenne * carra, le tout avec le sens de «pierre».

\section{Varia (Rohlfs, 55-59)}

85 : Gasc. agòr 'automne'. Gòrra (ALG III, 839) est attesté en Haut-Médoc et à Lesparre (Viaut 1999). Il se rattache à l'aire gasconne occidentale jusqu'à Soustons, une aire pyrénéenne abòr s'étendant sur le Béarn et la Bigorre. En outre, en Médoc, gòrra a en plus le sens d'herbe sèche d'automne (Listrac-Médoc). Rohlfs renvoie au basque agor avec les sens de «sec», «août», «septembre» selon les régions.

87 : Gasc. bisquèro 'grosse poutre qui soutient le faîte'. Le mot biscarret («ligne diagonale dans le tracé d'une parcelle destinée à être complantée de ceps de vigne») en Médoc (Viaut 1992, 111) peut-il se rattacher à cette motivation? Peut-être en identifiant le sème de ce qui est divisoire (Ravier 1992, 10) ? La base euskarienne bizkarr (dos, faîte de toit) est naturellement indiquée ici.

95 : Gasc. Chourrupà/hourrupà 'boire par gorgée, boire avec avidité'. Pour cela, en Médoc, (Viaut 1999), harlupar : «boire, aspirer goulument»; en Pays de Buch (Vidal 2002, 41), horrupar: «boire en aspirant, lapper». De même, en Bazadais (Vigneau 1879), hurlupal hourlupa/hourrupa : «boire, avaler avec bruit, en aspirant». Le basque hurrupatu (prendre des gorgées d'un liquide) est aussi indiqué (Lhande 1926).

111 : Laire de cabelh (épi), dérivé de cap (tête), avec la même motivation que le basque actuel buru (tête et épi) couvre intégralement le domaine linguistique gascon du nord au sud.

Dans le chapitre qui suit, «Le vocabulaire gascon comparé au vocabulaire espagnol», la pointe septentrionale du domaine gascon s'intègre bien à l'aire de concordance entre le gascon et les idiomes de l'Espagne septentrionale. L'origine de ces termes auxquels Edouard Bourciez s'était également intéressé (Bourciez 1901), est parfois obscure. Certains de ceux qui ont été retenus par Rohlfs n'ont pas d'équivalents en Bordelais ou bien connaissent une 
forme plus clairement pan-occitane comme c'est le cas pour l'entrée 117 : arremugà 'ruminer' (<* rimugare). On trouve romigar en Médoc, ou arromigar (sous «arroumiga») en Pays de Buch (Moureau 1870) et en Bazadais (Boisgontier \& Marquette 1982).

Pour le reste, nous pouvons signaler les attestations qui suivent, valables pour le Bordelais en général ou pour le Médoc en particulier. 61-72)

6. Concordance entre le gascon et les idiomes de l'Espagne septentrionale (Rohlfs,

123 : Gasc. Aujam, aujami 'bête de petite taille, ...', est répandu partout en Bordelais (aujame).

130 : Gasc. beregnà 'vendanger' a aussi donné par contraction Vrenhar (vendanger). Cette forme du sud, qui remonte jusque dans les landes, vient concurrencer à Listrac-Médoc (enq. A. Viaut) vendeunhar/venduunhar (ALG II 498), qui occupe par ailleurs l'ensemble du Bordelais.

134 : Gasc. (Pays de Barèges) 'réunion de choses attachées ou enfilées', rejoint carrassa, qui est courant en Bordelais, y compris en français régional vinicole. Ce substantif s'est surtout spécialisé pour désigner la rangée de barriques empilée en hauteur. Le dérivé verbal encarrassar (gerber, empiler en les rangeant, si possible en ligne, des barriques ou d'autres objets lourds et de même forme). On le trouve aussi en français régional dans cette contrée (Boisgontier 1991).

140 : Dit (doigt), au lieu de «det», ailleurs en occitan, est général en gascon.

141 : Entà (vers), est répandu jusqu'en Haut-Médoc inclus.

146 : Laire de gausar (oser) va jusqu'au Haut-Médoc inclus (ausar au nord) (ALG III 1082).

147 : Gorso 'précipice, ravin'. Le mot gòrça est répandu dans la toponymie du Bordelais et au-delà jusqu'en Limousin où il a aussi le sens de hallier dans la langue actuelle. Il semble qu'il faille plutôt lui retenir l'étymon gaulois *gŏrtia (haie), proposé dans le FEW (IV, 200). On retrouve d'ailleurs encore ce mot en Bas-Médoc (Talais, Saint-Vivien) sous gòrts (pl.) qui désigne un système de pêche fait de haies de pieux, serrés les uns contre les autres, destinées à piéger le poisson lorsque la marée descend. Il a également été transmis au singulier, gòrt, sur cette rive gauche de l'estuaire de la Gironde, comme désignant tout épi installé pour ralentir le courant et protéger la berge.

150 : Gasc. guit 'canard'. Guit est courant en Bordelais et s'inscrit dans une aire gasconne significative.

153 : Anc. béarnais fenar (Levy) 'prairie où l'on recueille du foin'. Henar est présent jusqu'à présent en Pays de Buch et Haut-Médoc.

155 : Gasc. (dans les Landes) hurre 'détermination, entrain'. Ladjectif hurègle, -a est répandu en Médoc avec le sens de «sauvage, farouche, agressif». Heruch, en Bas-Médoc a le sens de «sauvage, ce qui est sauvage» (Conord). Ce sens de farouche se trouve aussi dans le catalan furro ainsi que le note Rohlfs.

158: Gasc. limàc 'escargot'. Limac remonte du sud vers le Bordelais jusqu'à la lisière méridionale incluse du Médoc (ALG I 67). Cagolha, notamment, le concurrence ailleurs dans cette aire.

172 : Gasc. poupo 'mamelle'. Popa (sein) est répandu en Bordelais.

184 : Gasc. tourròc 'motte de terre'. La forme Turre est sélectionnée parmi d'autres pour la Gironde. On la trouve, en effet,en pays de Buch (Moureau 1870) avec le sens de «grand tas» 
mais ce mot relève-t-il vraiment du latin tŭrris (tour) (FEW, XIII 2, 435)?

186: Gasc. truc 'grosse sonnaille que porte le bélier', «d'origine onomatopéique», se trouve sous des formes très proches dans le Pays de Buch avec truque (Moureau 1870) ou truca et trunca/tronca, avec féminin à valeur augmentative) en Médoc (Conord). Le sens général de sonnaille pour le bétail est donné à ces derniers mots.

\section{Mots ibéroromans dont l'aire s'étend jusqu'en Gascogne (Rohlfs, 72-87)}

Ici, nous noterons directement après chaque numéro d'entrée, en privilégiant les attestations issues du Médoc, sachant que c'est de là que nous provient le plus d'informations, et que les formes dont elles témoignent sont le plus souvent dans le continuum aréal du gascon maritime.

197 : Rolha, rolhòt (petit fossé, drain), est général en Médoc. La forme rèlha, relhòt (idem), la concurrence en Bas-Médoc

201 : Vagant (paresseux, vaurien), est général en Médoc.

203: Braquin (soufflet de forge), est général en Médoc.

220: Chic (dans un chic: un peu) [tjik], [tsik], qui renvoie au basque txiki (petit), avec les diminutif et surdiminutif chicòt, et chicotet, est commun en Médoc. Le dérivé chicòi (petit enfant) est présent en Haut-Médoc comme dans une grande partie de la Gascogne lingistique.

223: Cohen, coheniu (ruche, rucher) sont les formes du Médoc à côté de bornac (ruche).

231 : Deishar (laisser) occupe le Haut-Médoc), et quitar le Bas-Médoc (ALG IV 1581).

236 : hart, -a (rassasié, -e) est général en Médoc et Bordelais, dans un grand continuum gascon.

238 : Le Médoc connaît comme continuateurs de Fageus (hêtre) : pin hag [pĩ hats] en Bas-Médoc, et pin hachèir [pĩ ha'tjej] en Haut-Médoc, qui désignent le pin parasol.

243 : La forme hitge (foie) est présente jusqu'au niveau de Bordeaux (Pessac et SaintAndré-de-Cubzac) (ALG IV 1186). Elle est relayée par hetge, présent sur une large bande garonnaise jusqu'à la pointe du Médoc.

260 : Soit pour la meule de foin, soit pour le tas de foin sur le pré, matòca (meule de foin) se dit en Médoc (ALG II 346 : Castelnau) et a été relevé à Listrac-Médoc (enq. A. Viaut). Madòca (tas de foin sur le pré) (ALG 342 : St-Yzans-Médoc, Hourtin, Cissac-Médoc) et matòc (idem) (ALG 342 : Castelnau), noté aussi à Listrac-Médoc (enq. A. Viaut), continuent le latin meta.

263 : Monaca (poupée), est général en Bordelais.

266 : Nina de l'uelh (pupille) (ALG IV 1440), répandu en Médoc et Bordelais.

268 : Nòtz, avec [0] (noix), contre [u] dans d'autres aires non gasconnes, est général.

272 : Pabiu (voir aussi : FEW VII 589) a été relevé en Médoc (Conord : noté pour la commune de Vensac) avec le sens précis de grosse mèche de filasse de chanvre trempée dans la résine et roulée pour faire des chandelles.

282 : Sarampion (rougeole), est commun là aussi.

284 : Secudir (secouer) est la forme prise en Médoc et Entre-Deux-Mers (ALG 1349).

289 : Só [su], bien que non attesté dans l'ALG en Bordelais, est très présent en Médoc, en synonymie avec sorelh. Il est attesté jusqu'au nord de la presqu'île (Conord) à côté aussi de sè $[\mathrm{s} \varepsilon]$. 
294 : Tòs, et les dérivés tòssa, tossòt, sont communs jusqu'en Bas-Médoc inclus (Conord) avec la signification de mangeoire (porc, chien). On le trouve également en Pays de Buch (Moureau 1870).

On fera abstraction de la partie concernant les «Mots galloromans, moins originale, dont l'aire s'étend jusqu'en Espagne du Nord» (Rohlfs, 87-96), la plus grande des entrées se retrouvant toutefois jusqu'en Bordelais.

\section{Les mots empruntés à l'espagnol (Rohlfs, 96-100)}

Nous nous permettrons d'ajouter : «ou d'origine commune avec l'espagnol». Moins productive que les autres listes pour le Bordelais, nous y voyons toutefois pour la zone la plus éloignée, le Médoc, de l'aire de l'espagnol, trois témoins intéressants qui paraissent davantage relever, pour nous, d'origines communes que d'emprunts sauf peut-être pour borric (cf. infra).

381 : Barren, barrena sont répandus dans la toponymie du Médoc et font partie de la langue transmise jusqu'à nous avec le sens de «bas-fond», dont un des synonymes est baisha, souvent francisé en une «vache», bien connue du cadastre girondin.

38 : Borric (âne), a été noté en Bas-Médoc (Gaillan) comme un mot à valeur hypocoristique pour ase et aine, communs en Médoc comme ailleurs.

389 : Pour l'espagnol charco (flaque d'eau), il semble bien que l'on puisse mettre en parallèle jarga et jargaud (Viaut 1998), signifiant la même chose. Le mot noté [dar'yaŭt] dans l'ALG (III, 783) au nord du Médoc, à Saint-Vivien-Médoc, paraît bien être jargaud prononcé en Bas-Médoc avec une affriquée sonore en attaque [dzardoulosaŭt].

\section{Les mots gascons (vocabulaire de mots rares et typiques) (Rohlfs, 101-105)}

Dans la liste de mots en graphie Gastou Febus qui figure sous le numéro 419, nous donnons les équivalents médoquins en graphie occitane à la suite de chaque entrée concernant cette aire médoquine incluant comme précédemment les points de Saint-Jean-d'Illac et Pessac. Les traductions entre parenthèses qui suivent l'attestation médoquine sont celles qui sont en usage dans la presqu'île. Elles ne sont que très rarement différentes de celles que Rohlfs avait transcrites. Nous faisons précéder certaines de ces entrées d'une astérisque rappelant que celles-ci sont déjà connues pour être communes dans tout l'ensemble du Médoc et, parfois, au-delà.

Arique $=$ arica (teille, déchet de la tige de chanvre) .

Arrounçà = ronçar (lancer, jeter). Répandu en languedocien aussi (Alibert 1966).

Aubisco $=$ aubesc (molinie).

Bade $=$ vader (naître, pousser, croître).

*Balanque = balanca (branche).

Barë = barra (courtilière), qui s'inscrit dans une grande bande du gascon martime, de la pointe du Médoc au Bas-Adour (ALG I, 53).

Bastë = basta (buisson épineux) Bas-Médoc : Vensac)

* Bèuc = bèuc (épine, arête de poisson).

Biganoùng = biganon (sarcelle d'hiver). 
Braude $=$ braude + embraudicar (boue, couvrir de boue, embourber) .

Bròs $=$ bròs (charrette forestière à deux roues).

Chaupà/tyaupì = chaupir (piétiner).

Chirpou $=$ chirp (lande du Haut-Médoc) .

Clòt $=$ clòt (trou d'eau, mare) (FEW II 796 préroman).

Còhou/còhe/còho = avec, pour le Médoc, les formes et sens particuliers: còha (coiffe, toile d'araignée), coheta (bonnet de nuit), et cohard/cohalh (traduit par «bonnet» en français local : grenier médoquin). Peut-être faudrait-il rattacher l'entrée 223 avec cohen (ruche) (cf. supra) à celle-ci?

Coudeyte $=$ codèita (bergeronnette), à Saint-Jean-d'Illac (ALG I, 28).

Craste $=$ crasta (fossé réalisé de main d'homme, fossé de drainage). Le Médoc landais continue l'aire du gascon maritime.

Creac $=$ creac

*Dibendres : divendres (orvet), avec deux aires compactes aux deux extrêmes du domaine gascon (Médoc et Comminges) (ALG II 39).

Eslambre $=$ eslombric (ALG III 821, Hourtin)

Estadi-s = estaïr (fatiguer).

Garbàtch = garbach/garbalha (ALG IV 1366, sud du Médoc)

Gaspo = gòspa (bale de céréale), à Listrac, Carcans, Saint-Laurent-Médoc (enq. A. Viaut). Voir aussi FEW (XXI 87).

Glabià = (?) clafità (mâchoire des animaux), synonyme, en outre, de maisherà, à SaintLaurent-Médoc (enq. A. Viaut).

Hourc $=$ horc (bois, bosquet).

Lajine = hlasina (étincelle). L'écriture «lajine» est une mauvaise interprétation, à notre sens, à partir du Palay car hlasina, en orthographe occitane moderne, et le mot proche «eslaside» dans la graphie» Gastou Fébus utilisée par Palay (1974), commencent en fait par FL- roman et la graphie phonétisante <esl-> transcrit simplement une des réalisations de /h/ initial en contact avec /// autrement regroupée avec d'autres ([ehl], [l]) sous la notation diasystématique et étymologique <hl> (Bec 1973, 171).

* Ledoune = ledona (arbouse) + ledonèir (arbousier) + vaca ledona (vache marine sauvage) + chivau ledon (cheval sauvage de la côte).

*Liroung = liron (couleuvre d'Esculape). Mot commun, y compris en français régional, en Bordelais.

Necère (nécessité) = necèira, dans l'expression «passar pas necèira de» (n'avoir de cesse de), employée en Bas-Médoc (Viaut 1998, 291).

*Padoeng = padoen (paturage commun).

Parròpi $=$ parròpia $($ paroisse $)$.

$*$ Sarc $=$ sarcle (houe).

Sèga $($ ronce, haie $)=$ sèga $($ haie $)$

Talòs (ver de terre) = (?) talòs (entrave de bois battant entre les pattes de l'animal), noté à Listrac-Médoc, Saint-Laurent-Médoc et Lamarque, en synonymie fréquemment avec talabòt. Talòs, avec cette signification, se retrouve ailleurs aussi en occitan et en catalan (Cf. talos, en catalan : morceau de bois épais).

Tuc, tuque = truc (tertre, hauteur). Cette forme se trouve en Médoc et Pays de Buch. 
Les listes de mots établies par Rohlfs ne pouvaient être plus exhaustives, pas plus que les attestations du nord du domaine gascon que nous avons repérées. Néanmoins, en vue de compléments ultérieurs et d'une approche plus affinée, nous ajoutons juste les quelques entrées suivantes. Elles ne résultent pas d'un choix raisonné mais découlent, à ce stade, de perceptions spontanées ou intuitives qu'il conviendra plus tard de réviser dans une perspective plus programmatique. En attendant, les mots présents en Médoc, et sélectionnés en suivant, nous rappelle en creux que le lexique des oiseaux et des poissons est finalement peu présent dans l'ouvrage de Rohlfs et qu'il gagnerait à être examiné de près.

Le mot chorra [tjurro] (ALG I, 26 : Pessac) désigne l'alouette. Deux autres attestations pour désigner ce même oiseau de l'ALG sont regroupées au centre du domaine gascon, à Saint-Justin et à Villeneuve dans l'est du département des landes : chorra e chorla. La même carte révèle par ailleurs les formes charla, à Beychac, près de Bordeaux, dans l'Entre-DeuxMers, et char [tjar], à Saint-Savin-de-Blaye, en zone d'oül de la Grande Gavacherie, marquée par les substrats occitans. Le mot est confirmé par le Glossaire de langue gabache de Charles Urgel (Urgel).

Pour le rouge-gorge, nous avons noté les mots gorrit à Cissac-Médoc (ALG IV, 1208 Cissac) et gorlit dans tout le centre du Médoc, attesté à Hourtin par l'ALG (IV, 1208) et recueilli à Lesparre (Conord), ainsi qu'à Saint-Laurent-Médoc, Listrac-Médoc, Avensan (enq. A. Viaut).

Dans ces deux cas, on pense aux termes basques txori (oiseau) et gorri (rouge), le groupe consonantique intervocalique -rr- trouvant fréquemment son doublon -rl- dans cette partie septentrionale du gascon. On l'a également pour sigòrra (ou sigòrla) (choin marisque, cf. supra) et pour ventòrra (ou ventòrla) (grand vent, tempête). Mais on l'a vu plus haut aussi pour horrupar/horlupar en Bazadais (cf. entrée $\mathrm{n}^{\circ}$ 95).

On se contentera de proposer enfin deux mots supplémentaires en leur adjoignant, comme hypothèse de liens possibles, des termes basques apparemment proches.

Ainsi de marmòta (hanneton) (ALG I 54) en Médoc, pour rester dans le règne animal. Ce mot est également connu en Blayais et dans le sud de la Saintonge (Urgel, Jônain). On retrouve marmòta de part et d'autre de la limite nord des départements de la Gironde et des Landes, et en Bas-Adour. Il est présent en basque, marmota (Lhande), qui l'a peut-être emprunté ou l'inverse, ainsi que dans les Pyrénées centrales gasconnes et le Val d'Aran (Tredòs). Lorigine du mot peut aussi être onomatopéique en songeant au bruissement fait par le hanneton en vol (?).

Osmic -ga (Listrac-Médoc, enq. A. Viaut) signifie: «embarrassant, énervant, en parlant d'un enfant qui se trouve toujours à l'embarras, qui touche à tout». Il paraît être propre au Médoc bien que cela reste encore à vérifier plus attentivement. Simple matériau à poser parmi les hypothèses : ozmin/ozpin en basque a le sens d'»insupportable» (Lhande).

De nombreux autres signifiants du «nord» pourraient également figurer dans cette liste de «mots rares et typiques» dont il conviendrait de revoir sans doute la conception. Le domaine maritime doit en fournir. Esquira (crevette), et colac (alose) ne sont point d'origine romane et ont leurs équivalents en basque (izkira, kolak). D'autres mots appellent l'attention, ne serait-ce que, par exemple haucha (raie pastenague) du Médoc et du Pays de Buch, etc.

Il résulte de cette première approche la formulation de l'hypothèse d'une place contradictoire de la pointe nord du domaine gascon par rapport à la conservation de traces lexicales spécifiques, voire anciennes. Lidée d'un amuïssement progressif vers l'est et le nord pourrait faire fi de possibilités de latéralisation potentielle dans toute aire dialectale, de la tendance archaïsante du gascon maritime, dont il participe souvent, et des zones îliennes et 
presqu'îliennes alors même que la zone prise en considération est bien en contact avec un pôle de diffusion actif des expressions linguistiques généralisantes et de la langue dominante la plus instrumentale.

\section{Références}

ALG < Séguy, Jean (1956-1973). Atlas linguistique et ethnographique de la Gascogne, avec la collaboration de J. Allières, X. Ravier et alii. Paris : CNRS.

Alibert, Louis (1966). Dictionnaire occitan-français d'après les parlers languedociens. Toulouse : Institut d'études occitanes.

Bec, Pierre (1968). Les interférences linguistiques entre gascon et languedocien dans les parlers du Comminges et du Couserans. Paris: Presses universitaires de France.

Bec, Pierre (1973). Manuel pratique de gascon moderne. Paris : A. \& J. Picard.

Boisgontier, Jacques (1991). Dictionnaire du français régional des Pays aquitains. Paris : Bonneton.

Bourciez, Édouard (1901). «Les mots gascons comparés aux mots espagnols». Bulletin hispanique, pp. 159-165, 226-233, 321-327.

Bouzet, Jean (1935). Compte-rendu de Gerhard Rohlfs. Le gascon. Études de philologie pyrénéenne. Bulletin hispanique, Vol. 39, n 1, pp. 79-85.

Chambon, Jean-Pierre (2003). «L'histoire linguistique de l'Aquitaine : de la romanisation à la fragmentation (1er-6e siècles). Éléments pour un modèle», in : Fray, J.-L. et Gorilovics, T. (dirs.). Regards croisés. Recherches en Lettres et en Histoire, France et Hongrie. Studia Romanica de Debreen, Bibliothèque française, $n^{\circ}$ 5, pp. 35-53.

Chambon, Jean-Pierre \& Greub Yan (2002). «Note sur l'âge du (proto)gascon». Revue de linguistique romane, Tome 66, n² 263-264, pp. 474-495.

Conord < Viaut, Alain (1999). «Le lexique bas-médoquin de François Conord». Revue des langues romanes, Tome CIII, n² , pp. 363-380.

Dinguirard, Jean-Claude (1977). «Aux origines du gascon». Travaux de linguistique et de littérature, XV, 1, pp. 243-244.

FEW < Wartburg, Walther von (1922-2002). Französisches Etymologisches Wörterbuch. Eine darstellung des galloromanischen sprachschatzes, 25 vol. Leipzig/Bonn/Bâle : Schroeder/ Klopp/Teubner/Helbing \& Lichtenhahn/Zbinden.

Gorrochategui Joaquín (s. d.). «Les études sur les langues anciennes des Pyrénées dans le sillage de G. Rohlfs, in : Lapport de Grhard Rohlfs (1892-1986) à la connaissance des langues pyrénéennes : parentés, disparités. Actes du colloque d'Oloron Sainte-Marie les 2 et 3 février 2007. Oloron Sainte-Marie : Mairie, pp. 9-30.

Jônain, Pierre A. (1869). Dictionnaire du patois saintongeais (2 fasc.). Royan : chez l'auteur.

Lhande, Pierre (1926). Dictionnaire basque-français et français-basque (dialectes labourdin, basnavarrais et souletin). Tome 1 Dictionnaire basque-français. Paris: Gabriel Beauchesne.

Moureau, Pierre (1870). Dictionnaire du patois de La Teste. La Teste: P. Moureau.

Palay, Simin (1974). Dictionnaire du gascon et du béarnais modernes. Paris : CNRS.

Ravier, Xavier (1991). «Occitan : les aires linguistiques», in : Lexikon der Romanistischen Linguistik, vol. V, 2, éd. par Holtus G., Metzeltin M. et alii. Tübingen : M. Niemeyer, 
pp. 80-105.

Ravier, Xavier (1992). «Préface» à : Viaut, A. Flor de vinha, en Médoc paroles d’oc du vignoble à Saint-Estèphe. Bordeaux: Maison des sciences de l'homme d'Aquitaine, pp. 9-10.

Région Aquitaine (2009). Enquête sociolinguistique Présence, pratiques et perceptions de la langue occitane, Bordeaux, Conseil régional d'Aquitaine, http://v4.aquitaine.fr/IMG/pdf/ Enquete_linguistique.pdf

Rohlfs, Gerhard (1977 [1935]). Le gascon : études de philologie pyrénéenne. Max Niemeyer ; Marrimpouey jeune : Tübingen, Pau.

Sauzet, Patrick (2006). «La spécificité phonétique du gascon, occitan extrême», in : Un pays dans sa langue», in : Le gascon dans l'ensemble d'Oc (Actes du colloque de Sabres, 9-10/10/2004). Sabres : Parc naturel régional des Landes de Gascogne, pp. 17-22.

Urgel Charles < Jagueneau, Liliane (éd.) (2014). Glossaire de langue gabache de Charles Urgel (1876-1947). Bordeaux: Maison des sciences de l'homme d'Aquitaine.

Viaut, Alain (1992). Flor de vinha, en Médoc paroles d'oc du vignoble à Saint-Estèphe. Bordeaux : Maison des sciences de l'homme d'Aquitaine.

Viaut, Alain (1998). Écrire pour parler. Los Tradinaires. Présentation d'une expérience d'écriture en occitan en Médoc. Bordeaux: Maison des sciences de l'homme d'Aquitaine.

Vidal, Yolande (1999). Dictionnaire gascon-français. Le parler du Bassin d'Arcachon et de ses environs. Bordeaux : Les Dossiers d'Aquitaine.

Vidal, Yolande (2002). Dictionnaire français-gascon. Le parler du Pays de Buch, Lo parlar deu Païs de Buch. D'hier à aujourd'hui. Bordeaux : Les Dossiers d'Aquitaine.

Vigneau, Bernard < Boisgontier Jacques \& Marquette Jean-Bernard (éds.) (1982). Lexique du gascon parlé dans le Bazadais 1879. Bazas : Les Cahiers du Bazadais. 